\title{
High Throughput Comparison of 13 Herbal Plants Extracted by Heat-Refluxed and Heat-Pressurized Extraction Techniques Using Chemometrics
}

\section{F. I. Abdullah}

Universiti Teknologi Malaysia

Lee Suan Chua ( $\sim$ chualeesuan@utm.my )

Universiti Teknologi Malaysia https://orcid.org/0000-0003-2493-9963

\section{Research}

Keywords: herbs, phytochemical, heat-refluxed extraction, heat-pressurized extraction, antioxidant, principle component analysis

Posted Date: October 16th, 2020

DOl: https://doi.org/10.21203/rs.3.rs-90768/v1

License: (c) (1) This work is licensed under a Creative Commons Attribution 4.0 International License.

Read Full License 


\section{Abstract}

\section{Background}

A reliable and effective extraction technique would be of great importance for high quality of herbal extracts. In particular, herbal extracts that are used for product development.

Method

Thirteen commonly used herbs of different families were selected and extracted using both heat-refluxed and heat-pressurized extraction methods for comparison. The yield and antioxidant capacity of the herbal extracts were compared and statistically analyzed for classification. Principal component analysis (PCA) was used to reduce the dimensionality of mass spectrometric data generated by LC-MS/MS.

Result

The extraction yield was found to be higher in heat-refluxed extracts than those prepared by heatpressurized system. Similarly, heat-refluxed system could produce herbal extracts with higher reducing power. Although majority of heat-pressurised extracts showed to have higher cation radical scavenging activity, the inhibition was mostly not more than $50 \%$. The result of PCA revealed that only $22.4 \%$ and $16.1 \%$ of the total variance was recorded for the first two components from the total of 41 and 38 components for heat-refluxed and heat-pressurized extracts, respectively. Although low similarity among phytochemicals, the herbal extracts still could cluster according to their antioxidant capacity rather than the extraction technique. Herbal extracts with close phytochemical profiles were also located in the similar cluster of antioxidant assay. This explains the importance of phytochemicals contributing to the antioxidant capacity.

\section{Conclusion}

To conclude, both extraction techniques were not comparable and the deviation of phytochemicals attributed to the difference in antioxidant capacity.

\section{Introduction}

Herbal medicinal plants are major sources of therapeutic phytochemicals that possess remarkable antioxidant capacity. The natural antioxidants have evoked considerable interest among nutritionists, food manufacturers and consumers because of their therapeutic potentials [1]. Therefore, a reliable and effective extraction technique would be of great importance for high quality of herbal extracts. The conventional extraction methods such as hot water bath, maceration, Soxhlet and percolation are very time consuming and require large quantity of solvents. Aside from extraction yield, the antioxidant capacity of samples was relatively low and poor [1]. This could be due to the degradation of nonthermolabile compounds after long period of solvent and heat exploration during extraction. There is an 
increasing demand for green extraction technique to shorten the extraction time and reduce solvent consumption without compensating the performance of extraction.

Although extraction technique and its operating variables seem to be the key parameters, the nature of raw materials is also affecting the quality of herbal extracts. Each herbal species has individual properties due to its unique physical structure and phytochemical profile. For instance, waxy, hairy and thick leaves may create an additional mass transfer resistance for phytochemical diffusion during extraction [2]. The setup of the extraction technology shall be applicable for a wide range of herbal material. Therefore, thirteen commonly available herbal leaves from different families were selected and gone through the similar process of extraction prior to high throughput screening for quality comparison. A hyphenated technique of LC-MS/MS was used to screen phytochemicals in the herbal extracts from both heat reflux and pressurized techniques. The operating variables of heat reflux system was converted and transferred to the heat pressurized system to have mimic extraction environment in both systems. The influence of extraction method on the yield, antioxidant capacity and the presence of marker compounds in the leaf samples was investigated. A multivariate pattern recognition approach, namely principal component analysis was also used to classify the samples based on their similarity and dissimilarity.

\section{Materials And Methods}

\section{1 Chemicals and herbal plants}

Ethanol (95\%) was purchased from Fisher Scientific Co. (Fair Lawn, New Jersey). HPLC grade of methanol, acetonitrile and formic acid were purchased from Merck (Darmstadt, Germany). 18.2 M $\Omega$-cm water was produced from Barnstead NANOpure Diamond water purification system (Thermo, Waltham, MA). 2,2'-azino-bis[3-ethylbenzothiazoline-6-sulfonic acid] (ABTS), ferric chloride hexahydrate and 2,4,6tripyridyl-s-triazine (TPTZ) were purchased from Sigma-Aldrich (St. Louis, MO). The 13 top priority herbs which are listed in the National Key Economic Area (NKEA) of Malaysia were selected and authenticated for their varieties, genus and family by the Unit Biodiversity, Institute of Bioscience, Universiti Putra Malaysia, Serdang, Selangor Darul Ehsan. All samples were dried and ground into fine powder before extraction.

\subsection{Phytochemical Extraction}

Phytochemical extraction was conducted by mixing $5 \mathrm{~g}$ powdered leaves with $70 \%$ ethanol $(250 \mathrm{~mL})$ in a round bottom flask and heat refluxed for 2 hours at boiling point $\left(85^{\circ} \mathrm{C}\right)$ under stirring condition. The extraction condition was then transferred equally to a smaller scale heat pressurized system to accelerate the extraction process [3]. The similar solvent-to-solid ratio of 50, $0.12 \mathrm{~g}$ powdered leaves were taken and mixed into $6 \mathrm{~mL}$ of $70 \%$ ethanol in a vial. The vial was then sealed and put into a Monowave 50 (Anton Paar, Austria) reactor to perform extraction. Based on the chart provided by the manufacturer, every $10^{\circ} \mathrm{C}$ increment in temperature can reduce half of the extraction time by 2 -fold rate acceleration in the closed 
vessel system according to the Arrhenius Law as presented in Eq. (1) [3]. Therefore, heat pressurized extraction was carried out at $115^{\circ} \mathrm{C}$ for $15 \mathrm{~min}$ at $800 \mathrm{rpm}$. After extraction, supernatant was centrifuged and filtered, and subsequently dried in an oven at $60 \mathbb{}$ C. The extraction yield of crude extract was recorded and stored at $-4^{\circ} \mathrm{C}$ until further analysis.

$$
k=A e^{\frac{-E_{a}}{R T}}
$$

Where $k$ is the rate constant, $T$ is the absolute temperature (in kelvin), $A$ is the pre-exponential factor, $E_{a}$ is the activation energy and $\mathrm{R}$ is the universal gas constant $\left(8.314 \times 10^{-3} \mathrm{~kJ} \mathrm{~mol}^{-1} \mathrm{~K}^{-1}\right)$.

\subsection{LC-MS/MS based data acquisition}

LC-MS/MS was performed using a Waters Acquity (Milford, MA) system coupled with a triple quadrupolelinear ion trap tandem mass spectrometer (Applied Biosystems 4000 Q TRAP; Life Technologies Corporation, Carlsbad, CA) and an electrospray ionisation (ESI) source. A C18 reserved phase Acquity column $(150 \times 4.6 \mathrm{~mm}, 1.7 \mu \mathrm{m})$ was used to detect the masses of phytochemicals in samples using negative ion mode. The mobile phase was a binary solvent system consisting of solvent $A$ (water with $0.1 \%$ formic acid) and solvent $B$ (acetonitrile). The gradient was as follows: $0-5 \mathrm{~min}, 10 \% \mathrm{~B} ; 5-15 \mathrm{~min}$, $10-90 \% \mathrm{~B} ; 15-20 \mathrm{~min}, 90 \% \mathrm{~B} ; 20-25 \mathrm{~min}, 90-10 \% \mathrm{~B} ; 25-30 \mathrm{~min}, 10 \% \mathrm{~B}$ for final washing and equilibration of the column for the next run. The flow rate was $0.20 \mathrm{~mL} / \mathrm{min}$ and the injection volume was $5 \mu \mathrm{L}$. All samples were filtered with $0.2-\mu \mathrm{m}$ nylon membrane filter prior to injection.

\subsection{Antioxidant activities}

The ferric reducing antioxidant power (FRAP) was determined according to the procedures described by Muthukrishnan et al. [4] with slight modification. A $100 \mu \mathrm{L}$ freshly prepared FRAP solution composed of $300 \mathrm{mM}$ acetate buffer $(25 \mathrm{~mL})$ at pH 3.6, $10 \mathrm{mM} \mathrm{TPTZ}(2.5 \mathrm{~mL})$ in $\mathrm{HCl}(40 \mathrm{~mL})$ and $20 \mathrm{mM}$ ferric chloride solution $(2.5 \mathrm{~mL})$ were mixed with different concentrations of crude extract $(100 \mu \mathrm{L})$ in a 96 -well plate. The absorbance of samples was read at $593 \mathrm{~nm}$. The reducing power was evaluated according to Eq. (2), and was compared to ascorbic acid as standard chemical. Triplicate experiments were carried out.

\section{Antioxidant capacity $\%=($ Asample - Acontrol $) /$ Asample $x 100$}

Another antioxidant assay based on the scavenging activity was performed using radical ABTS cation $(\mathrm{ABTS} \cdot+)$ decoloration assay [5]. The ABTS + solution was prepared by mixing ABTS $(7 \mathrm{mM})$ with potassium persulfate $(2.45 \mathrm{mM})$ in a volume ratio of $1: 1$. The solution was stored in a dark place for $6 \mathrm{~h}$, and then diluted with ethanol till the absorbance of the solution was about $0.70 \pm 0.02$ at $734 \mathrm{~nm}$ before experiment. A $100 \mu \mathrm{L}$ of different concentrations of crude extract was mixed with $100 \mu \mathrm{L}$ ABTS + solution in a 96-well plate. The absorbance was recorded at $734 \mathrm{~nm}$ after $20 \mathrm{~min}$ of incubation. The affinity of 
sample to quench free radicals was evaluated according to Eq. (2), and compared to ascorbic acid as standard chemical. Triplicate experiments were also carried out.

\subsection{Statistical Analysis}

Analysis of variance (ANOVA) was used as a univariate statistical approach to compare significant different between the two extraction methods. Principle component analysis (PCA) with Pareto scaling was used as an unsupervised multivariate data analysis to classify the mass spectrometric data and the effective concentration at $50 \%$ inhibition $\left(\mathrm{EC}_{50}\right)$ of herbal extracts prepared from both heat-refluxed and pressurized extraction systems. The minimum spectral peak width and mass tolerance were set at 0.05 and $0.10 \mathrm{Da}$, respectively. The retention time tolerance was $0.5 \mathrm{~min}$ and the maximum number of peaks was 10,000 .

\section{Results}

\subsection{Phytochemical extraction}

In this study, both heat reflux and heat pressurized extraction methods were applied on 13 types of herbal leaves. The extraction yield of both extraction methods is compared as shown in Figure 1. The figure clearly shows that heat reflux extraction displays higher yield than heat pressurized extraction for all tested herbal leaves. The heat reflux extraction produced extraction yield ranged from $7.0 \%$ to $17.2 \%$. The highest yield of crude extract was obtained from the leaf samples of Morinda citrifolia, followed by Citrus hystrix and Ficus deltoidea. For the heat pressurized extraction, the yield of extraction ranged from $4.8 \%$ to $21.1 \%$. The highest yield of crude extract was obtained from the leaf samples of Morus alba, followed by $C$. hystrix and F. deltoidea.

From PCA analysis, the data clustered herbal extracts into 12 principal components (PCs). The total variance of the first two PCs $37.3 \%$. The score plot clearly shows heat-pressurized extracts are grouped into a cluster (A) at the negative region of PC1 as marked in red circle (Figure 2(a)). On the other hand, 4 refluxed extracts, namely $P$. amaryllifolius, S. crispa, P. minor and E. longifolia are located away from the other herbal extracts. The phytochemical profiles of refluxed extracts appeared to be more scatter than pressurized extracts.

The individual datasets were further analyzed using PCA. The results were clustered into 41 and 38 principal components for refluxed and pressurized extracts, respectively. The score plots explain only $22.4 \%$ and $16.1 \%$ of total variance for the first two PCs for refluxed and pressurized extracts, respectively. The increase of principal components with low percentage of the total variance was explainable as the extracts were from different species of herbal plants. The phytochemical profile of $F$. deltoidea is significantly different from other extracts prepared by heat-pressurized system (Figure 2(b)). The score plot of heat-refluxed extracts could sub-cluster into 3 small groups as indicated in Figure 2(c). The figure also explains that the phytochemical profile of $M$. koenigi is near to that of $C$. longa. 
In order to compare the quality of extract produced from both extraction methods, the presence of previously reported compounds from individual herbs was listed in Table 1 (supplementary). The table shows that $60 \%$ of listed compounds could be detected from the extracts produced by heat-pressurized technique, whereas only $35 \%$ of them was successfully identified from the extracts prepared by heatrefluxed system.

\subsection{Antioxidant capacity of herbal extracts}

The progress curves of spectrophotometric inhibition are presented in Figure 3 and the results of $\mathrm{EC}_{50}$ are showed in Figure 4. Overall, heat-refluxed system was able to produce plant extracts with higher antioxidant capacity than heat-pressurized extraction. This is because the $50 \%$ inhibition of plant extracts prepared by reflux extraction was lower which was about 1-3 times lower than those of heat-pressurized system, particularly for FRAP assay. In other words, antioxidative compounds in refluxed extracts were mostly having reducing power to follow electron transfer mechanism.

However, different observation was noticed for ABTS assay which hardly achieved $50 \%$ inhibition, even though the concentration of extracts was increased up to $2.5 \mathrm{mg} / \mathrm{mL}$. Most of the heat-refluxed extracts showed to have lower cation radical scavenging activity than those of heat-pressurized system. Figure 4(b) shows only 4 herbs, namely E. longifolia, S. crispa, P. macrocarpa and C. hystrix exhibiting better radical scavenging activity. The plant extracts were likely to be reducing agents than radical scavengers. Based on the Figure 4, the ranking of herbal extracts in term of their antioxidant capacity was almost similar either as reducing agents or radical scavengers. The top three highest antioxidative extracts were E. longifolia, Piper betle and P. minor, whereas the lowest three antioxidative extracts were Pandanus amaryllifolius, $S$. crispa and $M$. citrifolia in the present study.

Unsupervised principal component analysis has clustered the data of $\mathrm{EC}_{50}$ into four principal components. The data is grouped according to the antioxidant assays rather than extraction techniques for the first two components (Figure 5). The first two components have explained $97.5 \%$ of the total variance. The leaf extracts of $P$. amaryllifolius (PA) and S. crispa (SC) with lower antioxidant capacity (higher $\mathrm{EC}_{50}$ ) are located at the positive region of $\mathrm{PC} 1$, whereas the leaf extracts with higher antioxidant capacity are located at the negative region of PC2. The low antioxidant capacity of PA and SC can be related to their phytochemical profiles as both herbal extracts also show to be in another isolated cluster as presented in Figure 2(a).

\section{Discussion}

Although phytochemicals make up less than $10 \%$ of the plant matrix, the therapeutic application of phytochemicals is getting proven scientifically [6]. Hence, the process of phytochemical extraction plays a critical role to obtain high quality of herbal extract. Extraction is a process to leach plant metabolites which are usually bioactive plant constituents from cells into solution under the stipulated operating parameters. Considerable effort has been made by worldwide researchers to find an efficient extraction 
method to recover as maximum as possible the desirable phytochemicals. Efficiency refers to not only the yield of extraction, but also the potency or bioactivity of the obtained plant extract [7]. Nevertheless, it is also important to highlight that the quality of plant extracts is also strongly relied on the nature of plant origin.

The plants of M. citrifolia, C. hystrix and F. deltoidea are characterized by thick, hard and waxy leaves. Waxy leaf is a special characteristic of higher plant which is considered as a barrier of plants against uncontrolled water evaporation, mechanical damage, and insect attack. Hence, the extraction of waxy leaves typically involves the use of solvents like methanol and dichloromethane [8]. Possibly, fatty substances of waxy leaves were co-extracted by aqueous ethanol, and indirectly contributed to the high yield of crude extract.

Heat reflux extraction is a conventional method extracting phytochemicals from herbal plants. Extraction was carried out by heat transfer via the process of convection and conduction. The extractability of phytochemicals depends on the solubility of compounds into solvent in the presence of heat [9]. Temperature is the most critical parameter in the heat reflux extraction [10]. However, heat reflux extraction is not suitable for extracting thermolabile natural products. The efficiency of phytochemical extraction under heat reflux technique is also influenced by factors such as the solvent to herbal material ratio and its particle size, as well as extraction time [10]. The limitation of the method is relatively slow, large quantity solvent consumption, high energy consumption, low quality extract, and degradation of heat labile constituents $[7,11,12]$.

Therefore, heat pressurized extraction or known as autoclave-type reactor integrates both parameters; heat and pressure in which extraction can be carried out at temperature far above the boiling point of solvent, and considerably shortening extraction time. In the present study, heat pressurized extraction was carried out in a sealed vessel heated at $115^{\circ} \mathrm{C}$ and 20 bar by applying conductive heating principles [13]. This method is relatively easy and efficient to handle because no running water to cold solvent vapor during extraction as in the reflux system. Moreover, heat pressurized extraction involves low energy consumption due to short period of extraction time.

An unsupervised multivariate data analysis was carried out on the mass spectrometric data of herbal extracts prepared by both heat reflux and pressurized techniques. PCA was used to classify and reduce the dimensionality of data for better visual inspection. It is a useful statistical pattern recognition tool, especially for highly complex datasets of natural product samples.

Two common antioxidant assays such as FRAP and ABTS radical cation scavenging, were also carried out to estimate the antioxidant capacity of herbal extracts. Both assays follow different mechanisms of antioxidation, namely electron transfer in FRAP assay and hydrogen atom transfer in ABTS assay.

Regardless the extraction technique, the 13 herbal extracts were prone to be electron donors as proven in FRAP assay. Often, polyphenols and stilbenes could be good electron donors, while phenolic acids could likely be hydrogen atom donors [14]. Plant polyphenols are multifunctional in the sense that they can act 
as reducing agents, hydrogen atom donors, and singlet oxygen scavengers. The antioxidative phenolics are converted into phenoxyl radical species which are more stable due to the electron delocalization within adjacent benzene rings or dimerization

\section{Conclusion}

The technique of extraction and its operating variables can affect the quality of herbal extracts in term of their phytochemical profiles, and consequently their antioxidant capacity. The present study proved that the heat-refluxed extraction technique was slightly better than the heat-pressurized system, in term of extraction yield and reducing power. The selected 13 herbs were likely to be reducing agents rather than radical scavengers.

\section{Declarations}

\section{Acknowledgement}

Not applicable

\section{Authors' contributions}

FIA wrote the manuscript and investigation. FIA and LSC systemically revised the manuscript for important content. FIA and LSC completed the figures and tables, collected literature and checked data, proposed the conception and designed the structure of the manuscript. All authors read and approved the final manuscript.

\section{Funding}

The authors would like to thank the financial support of Ministry of Higher Education and Universiti Teknologi Malaysia under the research grant of HICoE (4J263), and UTM-TDR 07G21 and UTMHR 08G84, respectively.

\section{Availability of data and materials}

Not applicable.

\section{Ethics approval and consent to participate}

Not applicable.

\section{Consent for publication}

The manuscript is approved by all authors for publication.

\section{Competing interests}


The authors declare that they have no competing interests.

\section{References}

1. Castro-López C, Ventura-Sobrevilla JM, González-Hernández MD, Rojas R, Ascacio-Valdés JA, Aguilar $\mathrm{CN}$, et al. Impact of extraction techniques on antioxidant capacities and phytochemical composition of polyphenol-rich extracts. Food Chem. 2017;237:1139-48.

2. Khanam S. Selection of suitable model for the supercritical fluid extraction of carrot seed oil: A parametric study. LWT. 2020;119:108815.

3. Anton Paar. Teaching guide Monowave 50. Anton Paar GmbH. 2018; p. 1-4. https://www.antonpaar.com/fileadmin/Landing-pages/Monowave_50_teaching_guide/Monowave50_Teaching_Guide_Preview.pdf

4. Muthukrishnan S, Manogaran P. Phytochemical analysis and free radical scavenging potential activity of Vetiveria zizanioides Linn. Int Res J Pharm. 2016;7(6):71-6.

5. Chua LS, Lau CH, Chew CY, Mohd Ismail NI, Soontorngun N. Phytomedicine Phytochemical profile of Orthosiphon aristatus extracts after storage: Rosmarinic acid and other caffeic acid derivatives. Phytomed. 2018;39:49-55.

6. Harbourne N, Marete E, Christophe J, Riordan DO. Analysis and Application 17 Conventional extraction techniques for phytochemicals. In: Tiwari BK, Brunton NP, Brennan CS, editors. Handbook of Plant Food Phytochemicals: Sources, Stability and Extraction. First. John Wiley \& Sons, Ltd; 2013. p. $400-10$.

7. Gupta A, Kothari V. Modern extraction methods for preparation of bioactive plant extracts. Int J Appl Nat Sci. 2014;1:8-26.

8. Ardenghi N, Mulch A, Pross J, Maria E. Organic Geochemistry Leaf wax $n$-alkane extraction: An optimised procedure. Org Geochem. 113. 2017;113:283-92.

9. Dhanani T, Shah S, Gajbhiye NA, Kumar S. Effect of extraction methods on yield, phytochemical constituents and antioxidant activity of Withania somnifera. Arabian J Chem. 2017;10:S1193-9. http://dx.doi.org/10.1016/j.arabjc.2013.02.015

10. Ngo YL, Lau CH, Chua LS. Review on rosmarinic acid extraction, fractionation and its anti-diabetic potential. Food Chem Toxicol. 2018;121(September):687-700.

11. Pouralinazar F, Aziz M, Yunus C, Zahedi G. Pressurized liquid extraction of Orthosiphon stamineus oil: Experimental and modeling studies. J Supercrit Fluids. 2012;62:88-95. http://dx.doi.org/10.1016/j.supflu.2011.12.009

12. Juangsamoot J, Ruangviriyachai $C$, Techawongstien S, Chanthai S. Determination of capsaicin and dihydrocapsaicin in some hot chilli varieties by RP-HPLC-PDA after magnetic stirring extraction and clean up with C18 cartridge. Int Food Res J. 2012;19(3):1217-26.

13. Kappe CO. My Twenty Years in Microwave Chemistry: From Kitchen Ovens to Microwaves that aren't Microwaves. Chem Rec. 2019;19(1):15-39. 
Figures

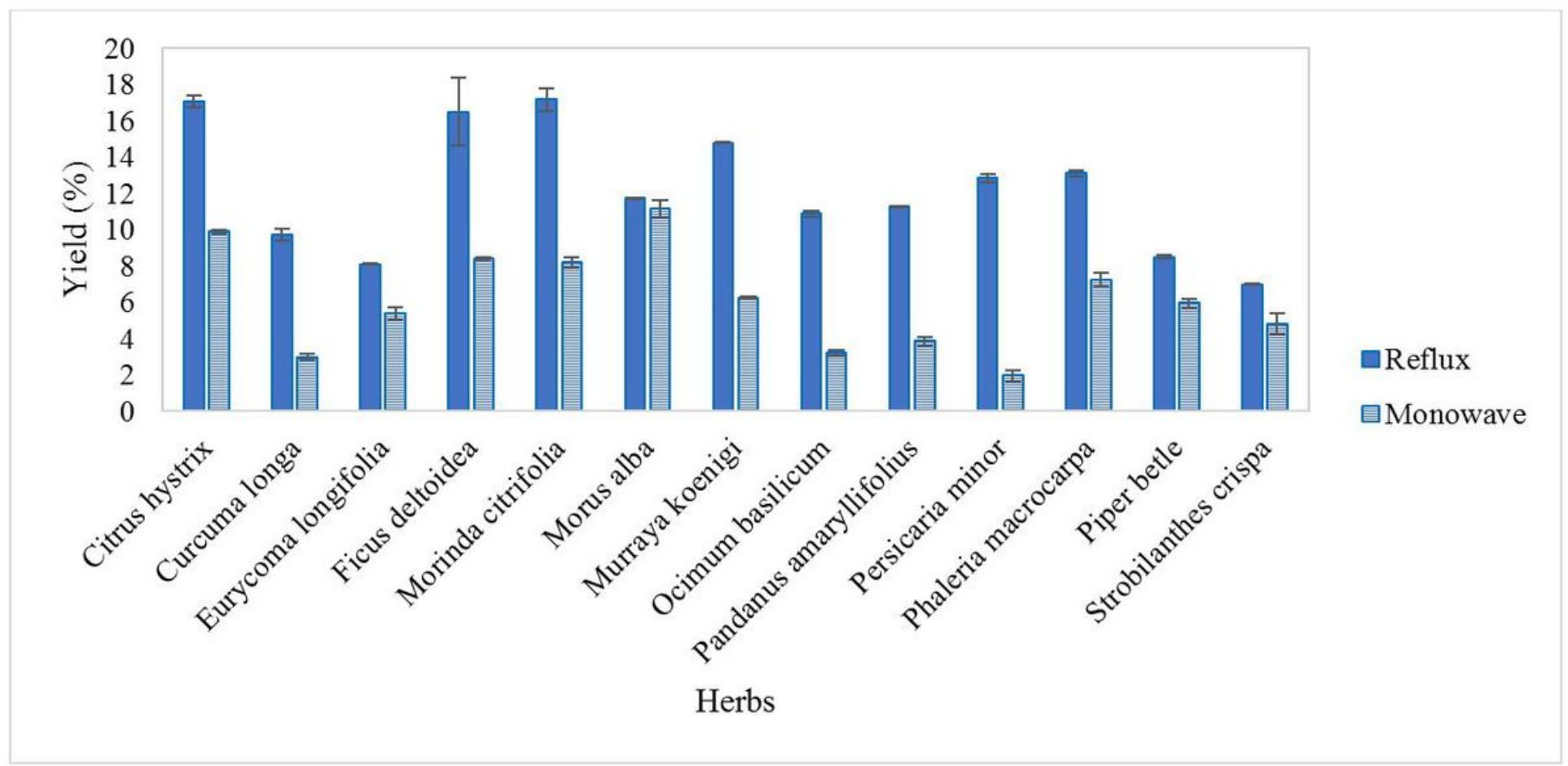

Figure 1

Extraction yield of both heat-refluxed (solid bar) and -pressurized (line bar) extraction for 13 types of herbal leaves 


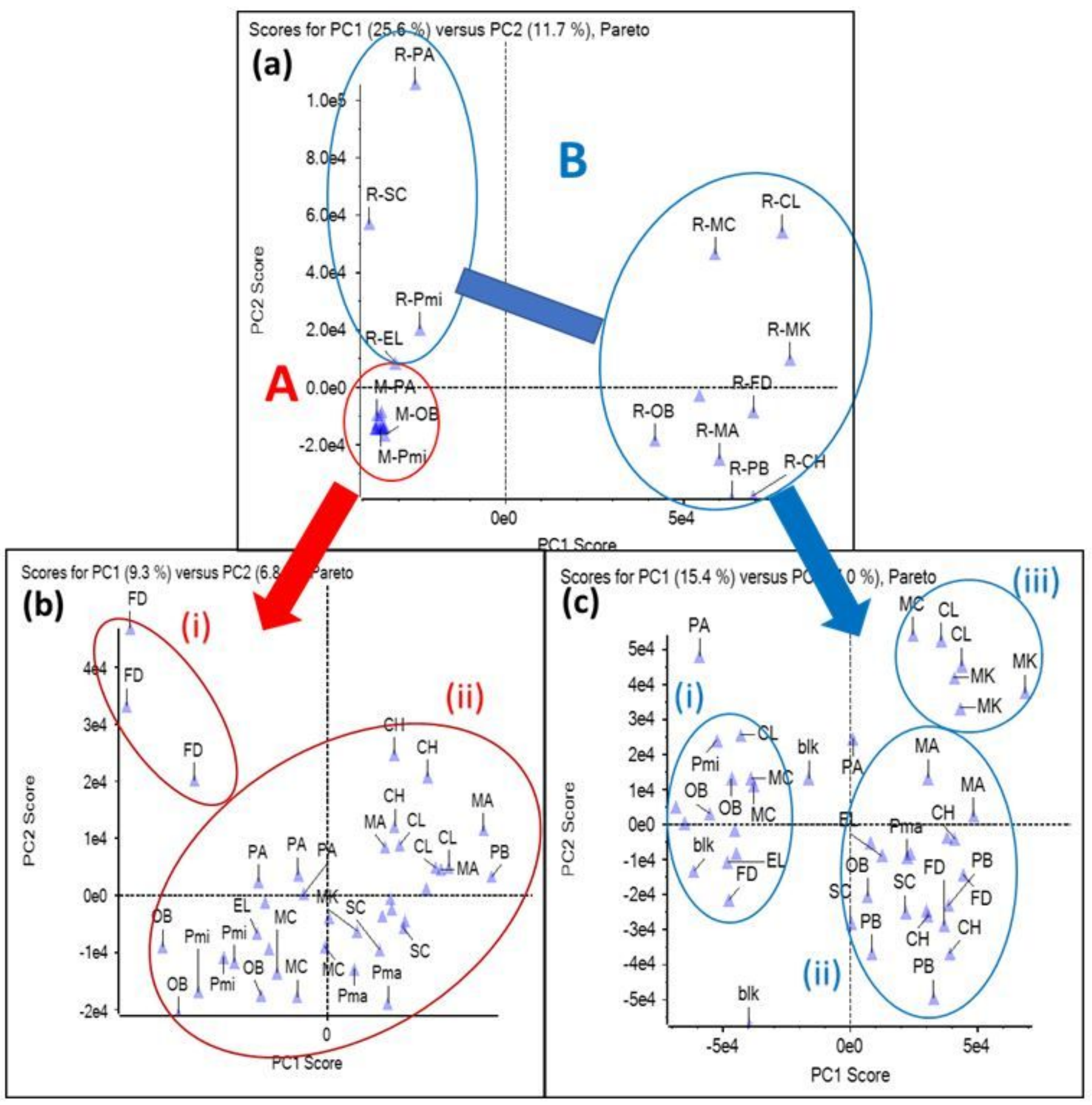

Figure 2

Score plots of 13 herbal extracts prepared by (a) both extraction techniques, (b) heat-pressurized system and (c) heat-refluxed system based on LC-MS/MS data 


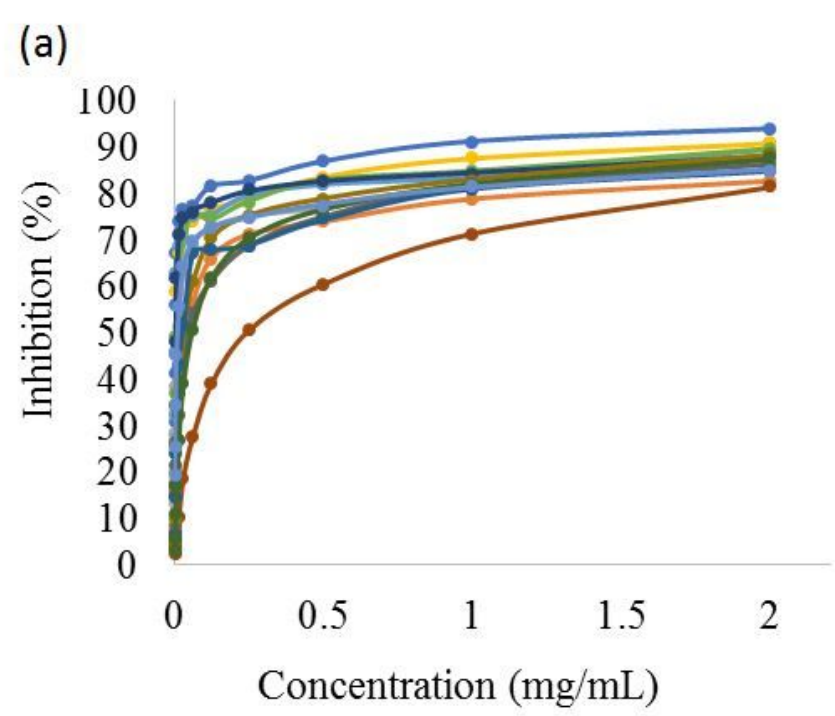

(c)

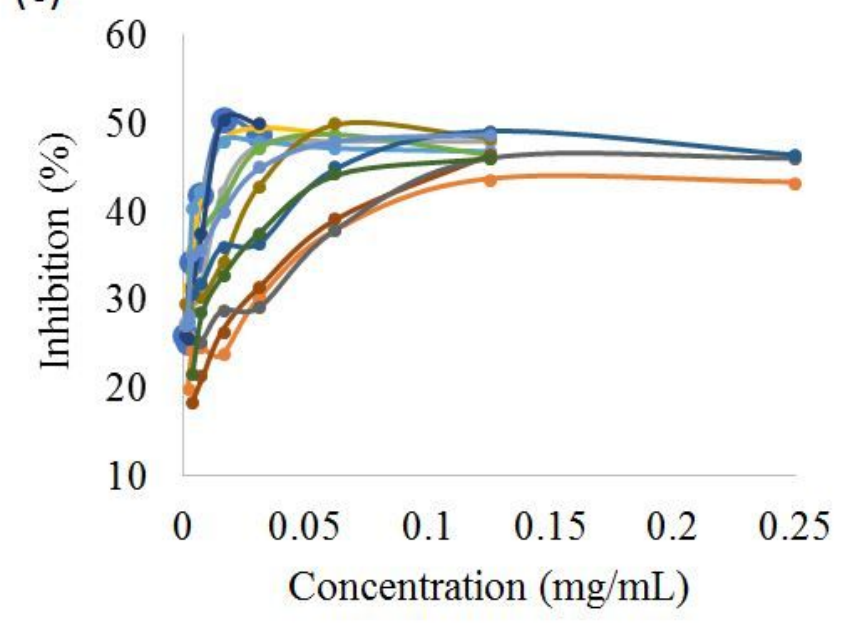

(b)

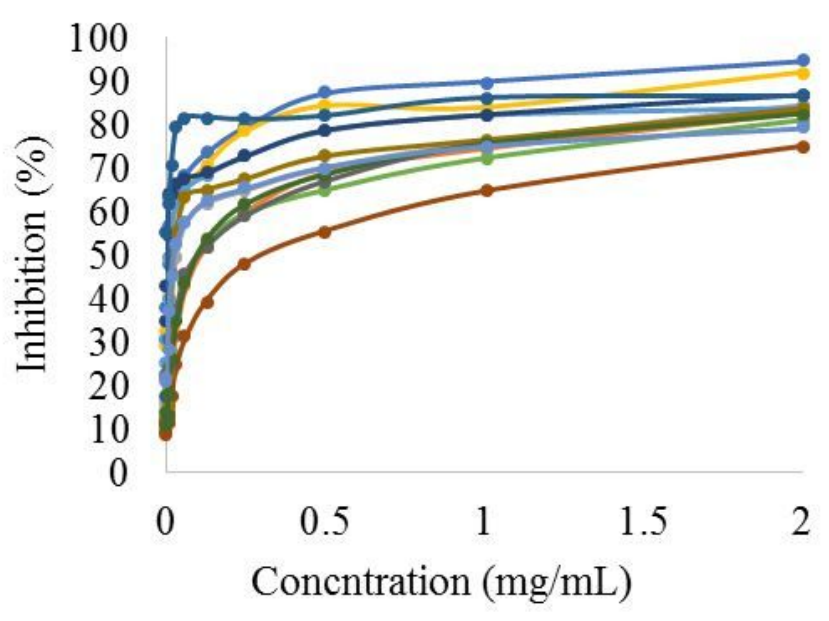

(d)

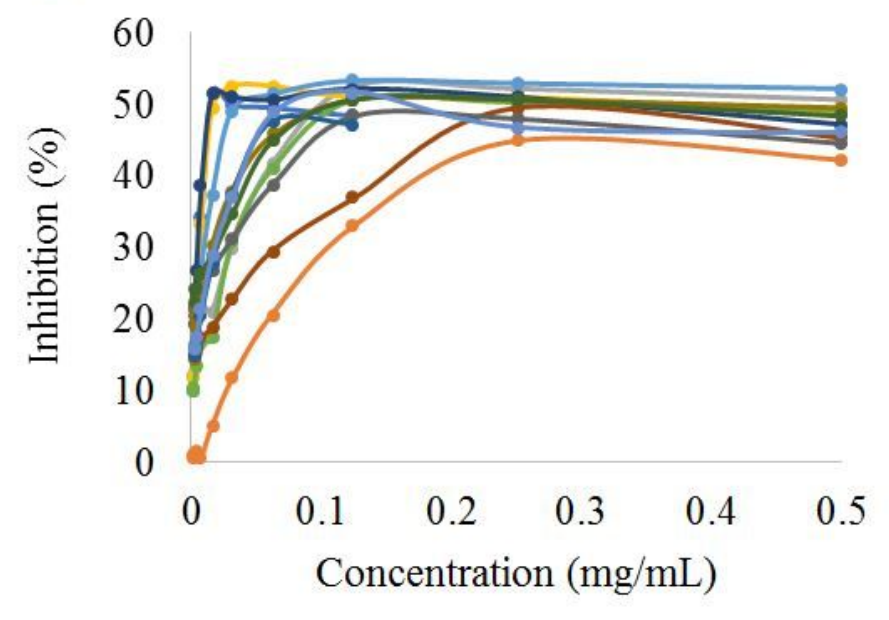

\section{Figure 3}

Progress curves of ferric reducing antioxidant power ( $a$ and $b$ ) and cation radical scavenging activity (c and d) using herbal extracts prepared by heat-refluxed ( $a$ and $c$ ) and -pressurized ( $b$ and d) extraction techniques, where Eurycoma longifolia (•); Pandanus amaryllifolius (•); Ficus deltoidea (•); Persicaria minor (•); Ocimum basilicum (•); Morus alba (•); Piper betle (•); Strobilanthes crispa (•); Morinda citrifolia $(\cdot)$; Phaleria macrocarpa $(\cdot)$; Curcuma longa $(\cdot)$; Citrus hystrix $(\cdot)$ and Murraya koenigi $(\cdot)$ 
(a) 300
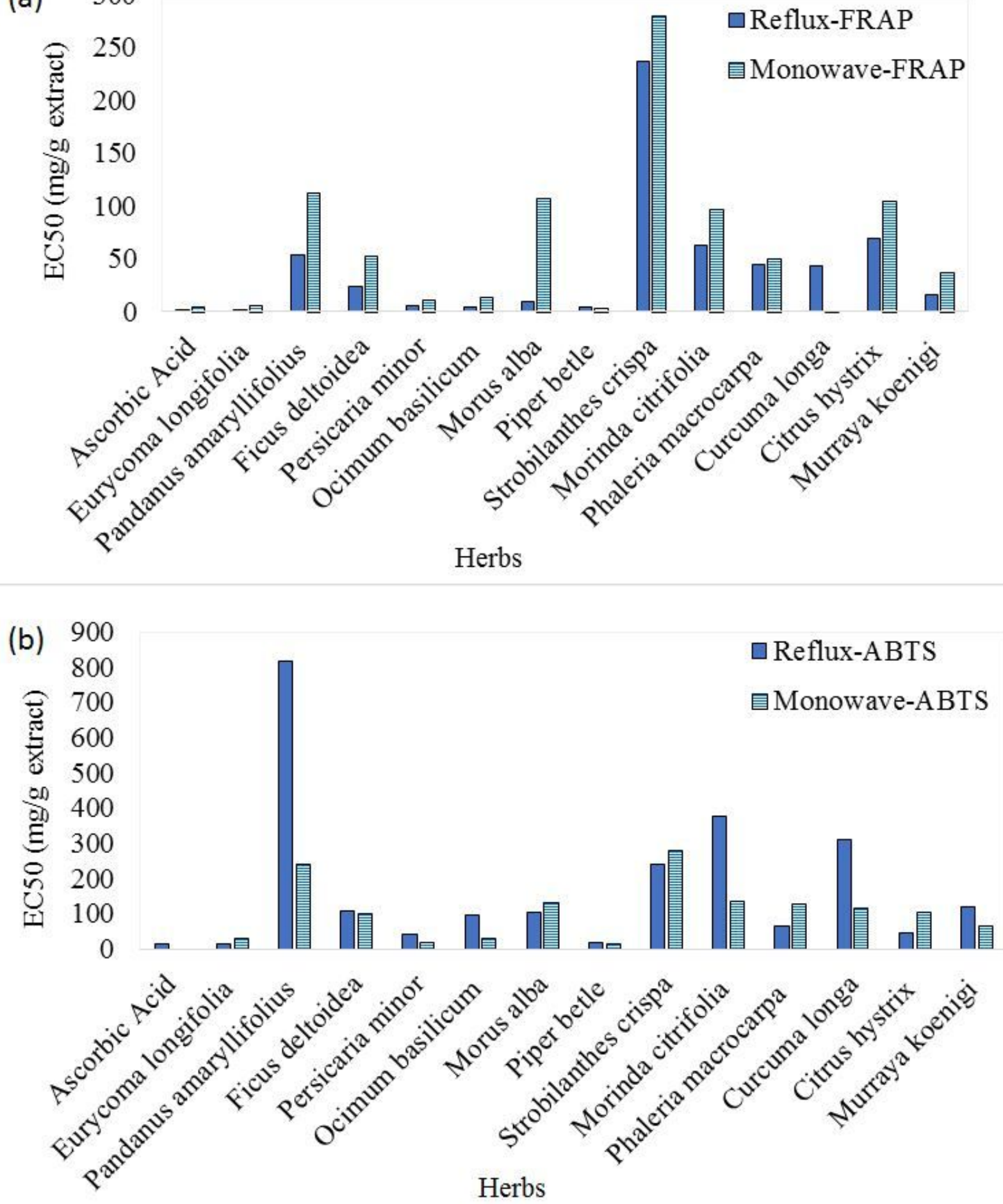

\section{Figure 4}

Antioxidant capacity expressed in EC50 (effective concentration at 50\% inhibition) for different herbal extracts using different extraction techniques, namely heat-refluxed (solid bar) and -pressurized (line bar) systems based on (a) FRAP and (b) ABTS assays 

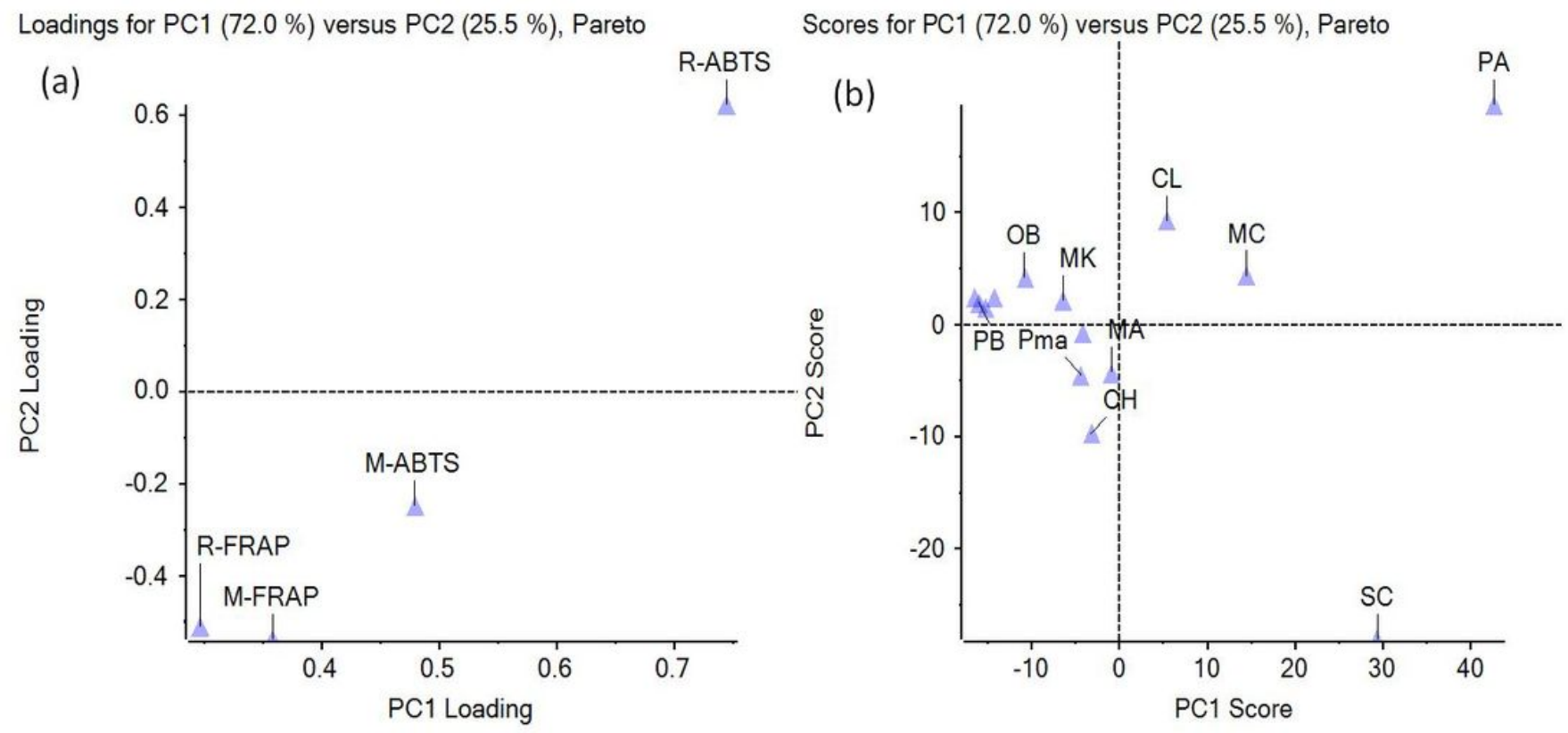

Figure 5

(a) Loading and (b) score plots of herbal extracts based on different extraction techniques and antioxidant assays for the first two principal components, where $\mathrm{R}$, heat-refluxed extraction; $\mathrm{M}$, heatpressurized extraction; FRAP, ferric reducing power and ABTS, cation radical scavenging activity.

\section{Supplementary Files}

This is a list of supplementary files associated with this preprint. Click to download.

- Table1suppl.c.docx 